\title{
Orestiada en O'Neill
}

\author{
María Lozano Mantecón* \\ «Cada cual recuerda bien a quien dio la \\ despedida, mas en vez de hombre, urnas y ce- \\ nizas, he ahi todo lo que volverá a vuestros \\ hogares). \\ La Orestíada : “Agamenón»
}

El héroe que vuelve de la guerra es, sin lugar a dudas, uno de los canales más típicos sobre los que se desarrolla la idea del regreso. El soldado que vuelve a casa, representa un módulo que se repite en todas las literaturas nacionales y cuyas características vamos a tratar de fijar.

El ejemplo más nítido que encontramos del tema, La Orestíada, nos muestra cómo el regreso del soldado no es sino un pretexto para expresar más que un retorno físico, una lección moral que estaría presidida por la desolación que sigue al sufrimiento. Veremos también cómo en la mayoría de las obras el regreso equivale a un punto de partida que se va a mostrar como la restauración del nivel inicial. Creo que hemos llegado a la palabra clave: restauración. En definitiva, la llegada del soldado equivale al punto cero del relato, al momento en que se va a pretender la vuelta al principio y la restauración de la situación inicial de antes de la guerra. Esta situación puede entenderse a dos niveles. Por un lado, tendremos la situación individual del héroe y su entorno, digamos, personal. De otro, la restauración de un nivel social y finalmente político.

La guerra va a presentarse como un paréntesis dentro de la trayectoria del héroe. Un paréntesis significativo, en cuanto que va a influenciar la línea de comportamiento anterior a la situación bélica y se va a manifestar en la práctica en un cambio de estructuras.

Volviendo a La Oestíada, encontraremos en ella el ejemplo más claro de cómo se efectúa esta restauración. Agamenón parte para la guerra como

* Depto. de Filología Inglesa. Universidad de Zaragoza. 
un héroe. Mientras, la línea principal de la tragedia continúa en la casa de los Atridas, y el puesto dejado vacante por el héroe viene a ser ocupado por Egisto, con lo cual no cabe duda de que se ha operado una sustitución a todos los niveles, no únicamente el personal. Paralelamente, la figura de Agamenón, que partió héroe, se degrada en su nuevo comportamiento. Ya no es el padre de los Atridas - al menos no cumple esa función en el palacio y en el lecho de Climtemnestra, - sino que las estructuras vigentes y la mentalidad le atribuyen precisamente las condiciones de un antihéroe, por su condición de cornudo. Su retorno, pues, ya no podrá ser una solución para la continuidad a la situación que dejó al partir, pues su degradación no le permite volver a ocupar su puesto. La solución será el asesinato, que aparece como la figura de una muerte ritual, de un holocausto, de un sacrificio.

A su vez, este retorno va a producir una serie de reacciones en cadena, que se van a presentar como una restauración de un nivel de justicia. Esto es, precisamente, lo que llevan a cabo los Atridas, Orestes y Electra.

Nos hallamos, pues, ante un tema eminentemente trágico, que nos enfrenta a la degradación de un héroe y a una restauración que nos haría pensar en tantas obras de Shakespeare ${ }^{1}$. En este sentido, el tema fue tratado por O'Neill en su trilogía Mourning Becomes Electra. Publicada en 1931, es, sin lugar a dudas, la Orestíada de los tiempos modernos, y el autor no dudó en exaltar los puntos de contacto entre ambas y «tipologizar» a la heroína en la Electra griega. Aunque tratemos fundamentalmente de fijar las características del héroe bélico en la narrativa americana, creo que sería interesante destacar unos cuantos puntos referentes a esta obra, ya que es el ejemplo más evidente del arquetipo, y luego pasaremos a considerar la degradación del mismo que se observa en la novelística.

1. Lo mismo en King Lear como en Richard II hay una imagen de humillación del monarca que lo mismo funde temas de la pasión de Cristo como de Everyman. Este (senequismo) isabelino con el que sufre Enrique IV, angustiado por la ausencia de su hijo, señala un camino donde la conquista de un orden familiar completo es meta necesaria. El hecho de que las tragedias de Shakespeare cierren un ciclo, sean como un difícil trecho entre el orden y el caos, añade todavía datos más insinuantes. Hasta en los niveles cómicos, donde el rey actúa de elemento taumatúrgico, hay un eco de piedad hacia quienes no pueden llegar a alcanzar la gloria. Observemos cómo lo entiende un crítico tan notable como Northrop Frye:

"Poetry must have an image: drama must have a character, and the feeling of lost social identity is what is expressed in the story of the fallen prince. The fallen prince is the "primal father» of a rather desparte myth of Freud'd, which seems to assume a crude notion of a (collective unconscious» that literary criticism, fortunately, does not need. In criticism, the murdered prince, from Agamennon onwards, stands for the sense of falling away from social unity which is constantly present in every generation": Fools of Time, by Northrop Frye, London: Oxford University Press, 1967, pp. 35-36.

Consúltese asimismo: What Happens in Hamlet, by John Dover Wilson, Cambridge: At the University Press, 1962, $357 \mathrm{pp}$; Shakespeare y la política, por Cándido Pérez Gállego, Madrid: Narcea, 1971, 246 pp. 
ORESTIADA EN O'NEILL

Estructuralmente, la tragedía de O'Neill consta de tres partes separadas: «Homecoming», «The Hunted» y «The Haunted», que corresponderian claramente a «Agamenón», «Las Coéforas» y «Las Euménides». Los caracteres cuyos comportamientos vamos a estudiar son Ezra Mannon y su esposa Christine (Agamenón y Climtemnestra), y sus hijos Orin y Lavinia Mannon( Orestes y Electra). El amante Adam Brant tendría su equivalente en el Egisto de Esquilo.

La acción se abre en abril de 1865, en una población de nueva Inglaterra, y más concretamente en el mundo cerrado que constituye «Mannon's House». La casa familiar va a transformarse en le espacio real de la tragedia. En ella el elemento femenino se prepara para la «llegada del guerrero». Superficialmente, todo haría pensar en la mujer-reposo del soldado. Como en su equivalente griega, el carácter mítico-trágico subyacente de las figuras centrales se ve contrarrestado por la presencia del coro, en este caso el pueblo llano, constituído por Seth, el «siervo fiel» y un grupo de amigos que penetran en el espacio Mannon y nos dan la visión exterior de la tragedia.

Pronto penetramos en el mecanismo interno de los personajes y establecemos claramente sus relaciones subyacentes que serán la causa de los comportamientos. Estos son la fijación materna de Orin y la paterna de Lavinia, o lo que es lo mismo, los complejos de Edipo y Electra, respectivamente. El matrimonio Mannon se había distanciado progresivamente hasta convertirse en extraños el uno para el otro, siendo los raíces de esta «mesentente» de origen sexual. Debido a ello, la hija mayor ejerce un efecto de repulsa sobre su madre quien la considera fruto de unas relaciones desagradables. Ella desarrolla, como consecuencia lógica, un afecto extralimitado por su padre. Por el contrario, la posición que Orin ocupa para su madre es la dejada vacante por el marido, Ezra, ya que su gestación y nacimiento tuvieron lugar en el lapso de tiempo en que Ezra Mannon abandonó su casa para marchar a la guerra de México, período en el que Christine se consideró por completo liberada de su esposo.

Hasta aquí, este complejo sistema de afectos invertidos se mantiene funcionando normalmente mientras sus sujetos se encuentran viviendo en el marco limitado de la casa Mannon. Sin embargo, O'Neill, en su obra, nos presenta una situación anormal en la que dos elementos integrantes del grupo han abandonado su espacio habitual. Su lugar dentro del mismo ha sido ocupado por un elemento extraño al conjunto, y así como Egisto ocupa el lugar de Agamenón en el lecho, un capitán de barco -Adam Brant- viene a llenar el lugar del marido de Christine. Y una vez más parece que $0^{\prime}$ Neill se esfuerza en seguir la pauta trazada por Esquilo: si Egisto pertenecía a la rama humillada de los Atridas, Adam Brante pertenece a la línea bastarda de los Mannon. De este modo, vemos cómo un 
elemento extraño se introduce en un mundo de relaciones establecidas y cerradas con un explícito deseo vengativo que tendría el efecto de una expiación moral. Pero el caso de Adam es más complejo, ya que no sólo desplaza a Ezra Mannon, el marido, objeto de su odio y su venganza, sino también a Orin, en su papel no de hijo, sino de amante, más virtual que real, de su madre.

En estas circunstancias, Lavinia se erige en juez y decide restablecer la situación de «Antes de la guerra» y preparar el marco del regreso de los Mannon, de forma tal que reencuentren el espacio y el ambiente que abandonaron al partir. Pero Vinnie no puede luchar contra la marcha de los acontecimientos, y su labor restauradora del orden viejo tiene consecuencias desastrosas, produciendo una serie de tragedias en cadena -muerte del padre, asesinato del amante, suicidio de la madre $y$, finalmente, del hermano- tragedias irreversibles como el propio paso del tiempo.

Ezra Mannon es un nuevo Agamenón volviendo victorioso del combate, aureolado de gloria y respeto por sus súbditos y espera encontrar la gratificación a través de un ritual en el que la mujer tiene que asumir el papel de protagonista, ritual fijo que se repite desde el rey Arturo volviendo a Guinevere, hasta el héroe de Joseph Heller abandonando la isla de Pianosa por el Paraíso sexual sueco. Sin embargo, Ezra muere asesinado por Climtemnestra Mannon. Su pretensión de recuperar el puesto abandonado, de regresar, en definitiva, precipita su fin. Su muerte debe ser entendida como una muerte ritual, porque si Ezra no se hubiera propuesto regresar -y por regresar entiendo siempre recuperar el puesto perdido, volver al punto inicial del que nos alejamos-, si no se hubiera muerto. Pero había iniciado muchos años antes un proceso divergente del de su mujer, y su lugar había sido ocupado por Orin, parcialmente, y por Adam Bront, posterior y totalmente, de tal modo que el regreso era imposible. El ya no tenía lugar como marido.

Orin es un caso semejante. Vuelve de la muerte - pues es su cara la que conoció en la guerra- y una vez más el objeto de su regreso es Christine. Partió contra sus deseos para hacerse un héroe ... «the day I set off to become an hero»- y vuelve como tantos otros convertido en un niño lleno de fantasmas y temores, con el único deseo de refugiarse en una madre que representa la infancia, el afecto y sobre todo la seguridad, la certeza de que nuevos fantasmas no van a turbar su mente. Llegar a casa supone la realización de un sueño rumiado muchas veces - «od, I've dreamed of coming home»-. El sueño, sin embargo, se resiste a tomar cuerpo real, y Orin no logra encontrar lo que dejó al partir.

Se encontraba inmerso en la muerte, en el absurdo de una situación - la guerra- que no había elegido por propia voluntad sino que le había 
ORESTIAADA EN O'NEILL

sido impuesta por su padre y por toda una tradición de Mannons, valerosos y conformes al orden establecido. Siendo niño, su padre le había arrastrado a la guerra para convertirlo en un hombre. $Y$, sin embargo, una vez en el campo de batalla, o lo que es lo mismo, en el campo de la vida, sólo ha encontrado muerte; y cuando un joven nos dice que

«I had the feeling that war meant murdering the same man over and over! and that in the end I would discover the man was myself! ${ }^{2}$.

significa que se ha elevado por encima de la circunstancia concreta y ha llegado al absurdo de una existencia en la que el hombre tiene que matar al hombre. Y Orin como Frederick Henry más tarde, y tantos otros, no ha entendido el lugar y función de esas ideas abstractas y rehusando enfrentarse a un problema ha recorrido al niño subyacente en todo adulto, buscando el Paraíso soñado donde la guerra es sustituída por el sexo. Orin sueña con las islas de paz de los marineros de Melville, y con su madre, con la doble función de mujer y de claustro en el que el niño se refugia cuando teme. Ha visto escenas terribles y sus fantasmas le perseguirán mientras viva.

La mujer, una vez más, tiende una trampa al guerrero. Orin, ante la evidencia de su madre Christine con su amante Adam, mata a éste último. Actúa movido única y exclusivamente por los celos, se siente sustituído por otro, y es esta imposibilidad de regresar a su antiguo puesto la que le mueve a la acción. En su asesinato a sangre fría mata simbólicamente, no a Adam Brant, sino al «amante» de su madre, y esta es la razón por la que el cadáver adquiere los rasgos de su padre, convirtiéndose en el prototipo del comportamiento de Orin en la guerra. Con lo cual ya no cabe duda de que la acción de Orin no ha sido sino una repetición de la de Adam Brant: el amante matando al que ha ocupado su puesto. Y de nuevo el esquema se repite. La gratificación buscada por Orin se le niega, es un nuevo padre buscando a la mujer-Christine, quien no pudiendo soportar la situación se suicida.

«Consumatum est» podríamos decir ahora. La tragedia ha terminado. Orestes ha hecho justicia en la casa de los Atridas, y Egisto y Climtemnestra yacen muertos en expiación por la suerte del padre Agamenón. Pero Esquilo dio una continuación a su obra y en las «Euménides» veíamos cómo las Furias perseguian incansables a Orestes, arrancándole de la casa familiar en un peregrinar interminable hasta el palacio de Atenea. Orin tampoco podía escapar a este destino $y$ «The Haunted» nos ofrece la visión de un joven perseguido por el fantasma de la culpa. Sabemos que

2. Mourning Becomes Electra, by Eugene O'Neill, London: Jonathan Cape, 1971, p. 156. 
Vinnie y Orin han emprendido el codiciado viaje por las Islas del Sur, y la tercera parte de la trilogía se abre con su regreso. Ya no es el regreso del soldado, sino el retorno del culpable, del vengador.

Han ocurrido demasiadas cosas en aquella casa para que la vida pueda restablecerse normalmente como si nada hubiera ocurrido. Los comportamientos habituales y las relaciones tradicionales se han quebrado y deben sustituirse por otros nuevos. $Y$ asi, por un proceso interesantísimo de mímesis Orin ha dejado de ser aquel joven que partió a la guerra. Ese ser ha sido destruído y en su lugar ha nacido el único posible, un nuevo Ezra Mannon. Y como tal ha producido en su hermana una nueva metamorfosis, de forma tal que Lavinia Mannon ha pasado a ser Christine Mannon, encontrando nuevos capitanes «Adam Brant» en los nativos de las Islas del Sur. La historia se repite, y Orin no puede sustraerse a su destino de Ezra Mannon enamorado de su mujer Christine-Lavinia:

«Can't you see I'm now in Father's place and you're Mother? That's the evil destiny out of the past I haven't dared predict. I'm the Mannon you're chained to! So isn't it plain?» ${ }^{3}$.

Ahora sí que podemos decir que de verdad han regresado. Están en el punto cero, en el punto de partida, en el que sus padres inconscientemente pusieron las bases de la tragedia. Sin embargo, la nueva Lavinia, como antes su madre, no responde, y Orin se suicida para encontrar definitivamente a la Mujer, la Isla de Paz:

«I love you now, with all the guilt in me. The guilt we share! Perhaps I love you too much, Vinnie!... That would be justice! Now you are Mother! She is speaking now through you! Yes! It's the way to peace_— to find her again_-my lost Island__- Death is an Island of peace, too» ${ }^{4}$.

Al llegar a este punto, la figura de Lavinia adquiere su verdadera dimensión como la real protagonista del drama, pues no ha sido otra, sino ella, el motor de todos los acontecimientos ocurridos. Ella fue quien descubrió a Adam Brant ysu pasado, ella quien enfrentándose a Christine en el primer acto, como afirmación de los Mannon ante los extraños, encendió el fuego de la tragedia. Ella es quien sola, decide afrontar el destino y hacer el sacrificio expiatorio en el lugar del drama, sin escapar hacia soluciones menos comprometidas como la de su hermano.

3. O'Neill, op. cit., p. 252.

4. O'Neill, op. eit., pp. 268 y 270. 
ORESTÍADA EN O'NEILL

«Don't be afraid, I'm not going the way Mother and Orin went. That's escaping punishment. And there's no one left to punish me. I'm the last Mannon. I've got to punish myself. Living alone here with the dead is a worse act of punishment than death or prison! I'Il never go out or see anyone! I'll live alone with the dead and keep their secrets and let them hound me, until the course is paid out and the last Mannon is let die! I know they will see to it I live for a long time! It takes the Mannons to punish themselves for being born! ${ }^{5}$

con una perspectiva total de la tragedia y de las interrelaciones entre sus caracteres, podemos establecer ya con bastante precisión los distintos niveles en los que se mueve, que si bien están magistralmente entrelazados unos con oitros, no por ello dejan de tener vigencia propia. Veremos cómo la estructura significante de la tragedia engloba más de un nivel de contenido:

En primer lugar, tenemos el nivel más superficial, el regreso del soldado. Este se nos revela como un retorno fallido, producido por lo absurdo de una situación político-social que destruye al individuo. Ya hemos visto cómo es Muerte lo que han encontrado en la guerra tanto Orin como su Padre, y cómo su actitud hacia la misma es el desprecio y el deseo de salir de ella y volver a la casa y la mujer. El absurdo de la guerra para el individuo es uno de los temas más antiguos de la Literatura Mundial, ya desde Homero, quien hizo argumento inicial de La Iliada la cólera de Aquiles por la esclava Criseida. En Literatura Americana, la guerra adquirió ya caracteres de farsa con Crane y su Red Badge of Courage, y los jóvenes decepcionados y de cierta manera castrados son legión, desde In Our Time de Hemingway, Soldier's Pay de Faulkner, Three Soldiers de John Dos Passos, etc., y lo absurdo de la contienda queda de manifiesto tanto en $A$ Farawell to Arms como en Catch-22. A esto se podría objetar la seriedad del concepto de frontera y de lucha manifiesto en las primeras obras americanas y en la guerra que sirve de telón de fondo a The Last of the Mohicans. A ello contestaremos que responde a una coyuntura en la que América se estaba formando como nación y el espíritu nuevo americano luchaba por existir y sobrevivir en un medio adverso. Es la gran epopeya de «The Making of America», de puritanos y caravanas, en la que el pueblo americano siente la necesidad de crear la épica de su nacimiento como lo hizo Roma y todos los pueblos. Hay que añadir también la influencia en esta literatura de la novela histórica europea respondiendo

5. O'Neill, op. cit., p. 287. 
a una mentalidad muy precisa ${ }^{6}$. Una vez la máquina político administrativa ha empezado a funcionar: una vez que que Estados Unidos ha entrado en la historia como nación, la epopeya se degrada y surge la novela, y con ella el intento del hombre de adecuación a un mundo que le ofrece guerras sin sentido en las que el individuo siente amenazada su personalidad por la gran máquina estatal productora de grandes ideas abstractas al servicio de diversas economías; y es ante esta situación de «nonsense» ante la que el individuo reacciona «ad absurdum».

Frente a la guerra el soldado se afirma a través del sexo y la mujer, y ello es ley en toda literatura y está respaldado incluso por leyes biológicas; por ello vemos cómo en literatura de guerra, el retorno equivale a retorno a la mujer, asociándola a un módulo de infidelidad. E incluso Joyce, que en su Llysses sigue paso a paso el pattern de La Odisea, introduce una variante al llegar a este punto. Mrs. Bloom contrasta con Penélope quien se caracteriza por la labioriosidad y fidelidad, la figura antagónica de Helena. Sin embargo, Molly Bloom recuerda más a la infiel esposa de Menelao y se define por dos notas contradictorias a las que definen a Penélope, como son la pereza más indolente y la infidelidad. Mientras Penélope termina convirtiéndose en el símbolo mediocre de la vida hogareña, Molly, por el contrario, se alza al nivel de símbolo básico del amor, entendido no como un exquisito sentimiento ni como un elemento ornamental, ni siquiera como una fuerza espiritual, sino que llega a ser algo más importante y básico, la cifra de una fuerza ideológica y biológica, fundamento de la existencia humana, la imagen de algo que podríamos llamar la tierra, la base estable de la existencia humana. $\mathrm{Y}$ así, algunos críticos han visto en ella la personificación del sí humano supremo, la aceptación de la vida y una contrafigura no sólo de Penélope, sino de la diosa Xea.

6. The Historical Novel, by Georg Lukacs, Harmondsworth: Penguin Books, 1963, $435 \mathrm{pp}$.

Esta tesis la deberíamos enfrentar con la que Steiner desarrolla sutilmente en The Death of Tragedy y así encontrar un plano donde se puede situar O'Neill en su propio territorio moral americano. La nueva Orestiada que presenta tiene mucho de mitología, de una venganza familiar contra las incursiones de los extraños. Hay un aire de rencor que señala Absalom, Absalom!, de Faulkner, y hasta deja al descubierto oue ese nuevo Hamlet se moverá con el código moral de los «outsider» de F. Scott Fitzgerald. Si para Lukacs la novela tiende a ser biografía, qué duda cabe que en O'Neill se consigue la epopeya de la lucha del hombre americano por descubrir su genealogía inédita. Una idea de Clifford Leach dará luz sobre esta hipótesis :

"Agammemnon was "King of Men», leader of the Greek Forces against Troy, ruler or Argos, Ezra Mannon was only a Brigadier-General in the Northern forces, and his house was one of many Greek-style residences in nineteenth-century New England. Behind the action of the Oresteia there was not only the general background of the Trojan War but the personal horror of Iphigenia's death, the savage conflict of the fathers of Agamemnon and Aegisthus, the history of a family that included Pelops and Tantalus and that had long known divine wrath": O'Neill, by Clifford Leach, Edinburgh: Oliver and Boyd, 1963, p. 88. 
ORESTIADA EN O'NEILL

Un segundo nivel que viene a sobreponerse al anterior lo encontramos en las relaciones familiares, que nos ofrecen la imposibilidad de unos afectos naturalmente ordenados, los cuales sobreviven gracias a sus desviaciones. Así, el regreso a la mujer se plantea como una lucha entre padre e hijo. Con lo cual, y si dejamos a un lado las excesivas asociaciones freudianas, tan pantentes en toda la obra de $\mathrm{O}^{\prime} \mathrm{Neill}$, nos llevaría a asociar la obra con un problema generacional. En el caso de Orin no cabe duda de que el enfrentamiento ha rebasado el plano teórico y ha modificado su comportamiento de forma trascendental, ya que le ha impuesto su participación en la guerra que ha conducido a ahogar su verdadera personalidad.

Junto a ello, las asociaciones padre-hija, madre-hijo, se presentan enormemente fecundadas en cuanto a sus consecuencias, constituyendo en sí mismas las causas de la acción. Lavinia interviene por amor a su padre y Orin mata por amor a su madre, esto es indudable y debemos decir que la progresión de esta doble tendencia invertida es la que proporciona el ambiente adecuado para la disociación del matrimonio. Aquí no podemos dejar de pensar en Hamlet y en cierta manera simpatizamos con las opiniones que realzan el afecto por su madre como primer motor de esa restauración del nivel de justicia que se lleva a cabo en la obra de Shakespeare.

Y llegamos a lo que - a mi modo de ver-constituye la estructura subyacente de la obra sobre la que reposan los niveles anteriormente expuestos. Observamos cómo toda la tragedia se desarrolla en un espacio cerrado - la casa de los Mannon- que en ocasiones llega a hacerse opresivo. Este espacio es un ambiente completo poblado por una serie de seres que ocupan todos los lugares del mismo, con posiciones muy definidas. Prueba de ello es que cuando tratan de salir de las mismas sobreviene la tragedia. Volviendo a la casa y observando fielmente las palabras de Christine :

«Each time I come back atter being away it appears more like a sepulchre! The «Whited» one of the Bible-pagan temple front stuck like a mask on Puritan grey ugliness! It was just like old Abe Mannon to build such a mostruosity -as a temple for his hatred!» ${ }^{7}$.

¿Un templo para el odio de los Mannon! Son graves estas palabras y sin embargo quizás de las más reales que pronuncie Christine. Este espacio, tiene un dueño absoluto, Ezra Mannon, y con él, todos los que llevan su apellido, quienes marcan estrictamente las fronteras fuera de las cuales se desarrolla el mundo de los demás, de afectos normales. Vemos cómo los sirvientes se introducen furtivamente en el jardín de la casa y cómo sus

7. O'Neill, op. cit., p. 34 . 
opiniones nos dan la pintura exterior de los Mannon, gente orgullosa, poderosa, que ha ocupado altos cargos en las estructuras que presiden la vida política del país. Ezra Mannon es muy rico, domina el comercio marítimo, es un alto magistrado de la justicia local y además gran general. Seth, el criado, es el pueblo fiel al dominante conocedor de la tragedia y de los inevitables fallos que encierra la blancura exterior de la casa Mannon, y sin embargo, los esconde, sólo porque Lavinia - una Mannon- se lo pide.

No creo que sea preciso ir más allá para darnos cuenta de que la estructura de la sociedad, o lo que es lo mismo, que se rige por sus mismas leyes: el poder y el respeto a la autoridad, el dinero, la separación total entre estamentos o clases y, presidiéndolo todo, la figura del tirano: Ezra Mannon. La casa de Mannon está asentada en tierra firme y, sin embargo, no presenta grandes diferencias con el Pequod, el barco donde el capitán Ahab imponía sú voluntad en búsqueda de la ballena blanca, Moby Dick. Tampoco es casualidad que los marineros de Melville huyeran a las islas Afortunadas, las mismas islas con las que sueñan Christine y Orin, mientras que Lavinia está fanatizada por la personalidad del padre, como estaban los contramaestres del Pequod por la de su capitán. Este Universo impone sus leyes que deben ser obedecidas tajantemente. Así, cuando años atrás un Mannon se salió de ellas enamorándose de la nodriza de su hermano y engendrándo un hijo, tuvo que abandonar la casa familiar y fue desterrado. Mientras tanto el Universo Mannon siguió funcionando.

Sobrevino la guerra, empresa a la que Ezra no podía faltar. Era un esquema más en su escalada de valores respetados; de este hecho se van a derivar consecuencias funestas y en el universo Mannon van a producirse graves roturas. Ya se nos dice que la guerra de México tuvo consecuencias importantes: la desviación del afecto de Christie hacia su hijo Orin; pero la actual, la guerra de Secesión, va a producir la caída del Dictador, su muerte y la destrucción de su Universo. ¿Cómo sobreviene? Mediante un proceso muy sencillo: La introducción de un elemento extraño al grupo, exterior a la sociedad cerrada que hasta entonces había guardado sus fronteras. Un elemento que, por otra parte, tiene derecho a pertenecer a esa sociedad cerrada, pues si Adam Brant es hijo de la sirvienta Mara Brantôme, no por eso deja de llevar el apellido y la sangre Mannon. Lo cierto es que introduce el caos y lleva a cabo su venganza por procedimientos no conformes a la ortodoxia Mannon. Estos no son sino la subversión de la propia estructura de esa sociedad cerrada sobre cuatro pilares fundamentales: Ezra-Christine-Orin-Lavinia. El proceso posterior era fácil. Minadas las bases, los odios al tirano, ocultos hasta entonces, suben a la superficie y así vemos cómo Orin y Christina expresan su deseo de salir de su influencia y huir hacia paraísos vegetales. $Y$ en este punto es donde entra en juego el nivel dos analizado anteriormente, los odios paterno-fi- 
liales unidos a los complejos sexuales, que precipitan la tragedia, de forma tal, que el espacio Mannon queda desprovisto de su dueño y Señor.

$\mathrm{O}^{\prime}$ Neill nos propone al final del drama la tesis de crimen y castigo, pues vemos cómo Lavinia se erige en víctima expiatoria de toda una sociedad cuyos pecados son anteriores a ella - tesis puritana- porque ¿habría tenido lugar la tragedia sin un universo que propugnara la expulsión de los padres de Adam? Unos héroes partieron a la guerra para encontrar a su vuelta una casa-sociedad deshecha, sus lugares-funciones dentro de ella ocupados por otros individuos, y como única salida se les presentó la muerte, y a través de ella, la expiación.

Hasta aquí hemos asistido al desarrollo de la tragedia. Si me he permitido recurrir a esta obra dramática a la hora de hallar unas posibles constantes que nos permitieran delimitar «el regreso del soldado» como una temática fija dentro de la literatura, ha sido porque me parecía un ejemplo perfecto de acomodación del mito clásico a una realidad nacional, la U. S. A. de Nueva Inglaterra, a la vez que nos iba a servir de referencia para observar la degradación que del mito se da en la narrativa, y al mismo tiempo, nos puede servir para establecer la diferenciación de niveles que se da en toda obra.

La literatura bélica norteamericana no es demasiado abundante. En principio, y debido a sus evidentes condicionamientos históricos, tendremos que prescindir del héroe bélico dentro de un tratamiento más o menos épico. Quizá en este sentido la obra de O'Neill nos haya sido enormemente útil para establecer el arquetipo. 
